\title{
COSMOLOGY AND SPACETIME SYMMETRIES
}

\author{
RALF LEHNERT \\ Department of Physics and Astronomy \\ Vanderbilt University, Nashville, Tennessee, 37235 \\ E-mail: ralf.lehnert@vanderbilt.edu
}

\begin{abstract}
Cosmological models often contain scalar fields, which can acquire global nonzero expectation values that change with the comoving time. Among the possible consequences of these scalar-field backgrounds, an accelerated cosmological expansion, varying couplings, and spacetime-symmetry violations have recently received considerable attention. This talk studies the interplay of these three key signatures of cosmologically varying scalars within a supergravity framework.
\end{abstract}

\section{Introduction}

Sizable efforts are currently directed towards the search for a more fundamental theory that must resolve a variety of theoretical issues left unaddressed in present-day physics. Many approaches along these lines require novel scalars as a key ingredient. Moreover, scalar fields are often invoked in cosmological models ${ }^{1,2,3}$ to explain certain phenomenological questions, such as the observed late-time accelerated expansion of the Universe, the horizon and the flatness problem, or the claimed variation of the fine-structure parameter. The search for observational consequences of such scalars can therefore yield valuable insight into new physics.

Scalar fields can acquire global expectation values that vary with the expansion of the Universe. Measurable effects of variations in the scalar background are typically feeble because of the cosmological time scales involved. This suggests ultrahigh-precision studies as a promising tool for experimental investigations. The remaining task is to identify suitable tests.

Varying scalars determine a spacetime-dependent background that violates translation invariance. This effect could be measurable because symmetries are typically amenable to high-precision tests. Moreover, translation-invariance breakdown is typically associated with Lorentz violation $^{4,5}$ since translations, rotations, and boosts are intertwined in the Poincaré group. This perhaps less appreciated result opens a door for al- 
ternative experimental investigations of cosmologically varying scalars and is the primary focus of this talk.

Lorentz and CPT breakdown has also been suggested in other contexts as a candidate Planck-scale signature including strings, ${ }^{6}$ spacetime foam,,${ }^{7,8}$ nontrivial spacetime topology, ${ }^{9}$ loop gravity, ${ }^{10}$ and noncommutative geometry. ${ }^{11}$ The emergent low-energy effects are described by the Standard-Model Extension (SME) ${ }^{12}$ which has provided the basis for studies of Lorentz and CPT breaking with mesons, ${ }^{13,14,15,16}$ baryons, ${ }^{17,18,19}$ electrons,${ }^{20,21,22}$ photons, ${ }^{23}$ muons,${ }^{24}$ neutrinos, ${ }^{12,25}$ and the Higgs. ${ }^{26}$

\section{Connection between translation and Lorentz symmetry}

Consider the angular-momentum tensor $J^{\mu \nu}=\int d^{3} x\left(\theta^{0 \mu} x^{\nu}-\theta^{0 \nu} x^{\mu}\right)$, which generates boosts and rotations. Its construction involves the energymomentum tensor $\theta^{\mu \nu}$, which is no longer conserved when spacetimetranslation symmetry is broken. Consequently, $J^{\mu \nu}$ will typically depend on time, so that the usual time-independent boost and rotation generators cease to exist. As a result, Lorentz and CPT invariance is no longer assured.

Lorentz violation through varying scalars can also be understood in a more intuitive way. A varying scalar is always associated with a nonzero 4 -gradient. This background gradient determines a preferred direction on scales comparable to the variation: Consider, for instance, a particle species that is coupled to such a gradient. The propagation features of these particles might now depend upon whether the motion is perpendicular or parallel to the background gradient. This implies physically inequivalent directions, and thus the violation of rotation invariance. Since rotations are contained in the Lorentz group, Lorentz symmetry must be broken.

We finally establish the effect at the Lagrangian level. Consider a model with a varying coupling $\xi(x)$ and two scalars $\phi$ and $\Phi$. Suppose that the Lagrangian contains a term $\xi(x) \partial^{\mu} \phi \partial_{\mu} \Phi$. We next integrate the action by parts with respect to the partial derivative acting on $\phi$. This produces an equivalent Lagrangian $\mathcal{L}^{\prime} \supset-K^{\mu} \phi \partial_{\mu} \Phi$. Here $K^{\mu} \equiv \partial^{\mu} \xi$ is a nondynamical background 4 -vector violating Lorentz invariance. Note that for cosmological variations of $\xi$ we have $K^{\mu}=$ const. on small scales.

\section{Toy supergravity cosmology}

We now illustrate the result from Sec. 2 with a toy model. This model is motivated by pure $N=4$ supergravity in four spacetime dimensions. Although unrealistic in detail, it is a limit of $N=1$ supergravity in eleven 
dimensions, which is contained in M-theory. One thus expects that our model can illuminate generic aspects of a candidate fundamental theory.

Suppose that only one of the model's graviphotons, $F^{\mu \nu}$, is excited. Then, the bosonic part of pure $N=4$ supergravity is given by 27,4

$$
\begin{array}{r}
\kappa \mathcal{L}_{\mathrm{sg}}=-\frac{1}{2} \sqrt{g} R+\sqrt{g}\left(\partial_{\mu} A \partial^{\mu} A+\partial_{\mu} B \partial^{\mu} B\right) / 4 B^{2} \\
-\frac{1}{4} \kappa \sqrt{g} M F_{\mu \nu} F^{\mu \nu}-\frac{1}{4} \kappa \sqrt{g} N F_{\mu \nu} \tilde{F}^{\mu \nu}
\end{array}
$$

where $M$ and $N$ are known functions of the scalars $A$ and $B$. The dual field-strength tensor is $\tilde{F}^{\mu \nu}=\varepsilon^{\mu \nu \rho \sigma} F_{\rho \sigma} / 2$ and $g=-\operatorname{det}\left(g_{\mu \nu}\right)$, as usual. Note that we can rescale $F^{\mu \nu} \rightarrow F^{\mu \nu} / \sqrt{\kappa}$ removing the explicit appearance of the gravitational coupling $\kappa$ from the equations of motion.

For phenomenological reasons, we also include $\delta \mathcal{L}=-\frac{1}{2} \sqrt{g}\left(m_{A}^{2} A^{2}+\right.$ $m_{B}^{2} B^{2}$ ) into $\mathcal{L}_{\mathrm{sg}} .{ }^{5}$ We further represent the model's fermions ${ }^{27}$ by the energy-momentum tensor of dust $T_{\mu \nu}=\rho u_{\mu} u_{\nu}$ describing, e.g., galaxies. Here, $u^{\mu}$ is a unit timelike vector and $\rho$ is the fermionic energy density.

The usual assumption of an isotropic homogeneous flat FriedmannRobertson-Walker Universe implies that $F^{\mu \nu}=0$ on large scales. Our cosmology is then governed by the Einstein equations and the equations of motion for the scalars $A$ and $B$. At tree level, the fermionic matter is not coupled to the scalars, so we can take $T_{\mu \nu}$ as covariantly conserved separately. Searching for solutions with this input yields a nontrivial dependence of $A$ and $B$ on the comoving time $t$.

\section{Lorentz violation}

Consider small localized excitations of $F_{\mu \nu}$ in the scalar background $A_{\mathrm{b}}$ and $B_{\mathrm{b}}$ from Sec. 3. Since experiments are usually confined to a small spacetime region, it is appropriate to work in local inertial coordinates. The effective Lagrangian $\mathcal{L}_{\text {cosm }}$ for such situations follows from Eq. (1) and is

$$
\mathcal{L}_{\mathrm{cosm}}=-\frac{1}{4} M_{\mathrm{b}} F_{\mu \nu} F^{\mu \nu}-\frac{1}{4} N_{\mathrm{b}} F_{\mu \nu} \tilde{F}^{\mu \nu},
$$

where $A_{\mathrm{b}}$ and $B_{\mathrm{b}}$ imply the time dependence of $M_{\mathrm{b}}$ and $N_{\mathrm{b}}$. Comparison with the usual Maxwell Lagrangian $\mathcal{L}_{\mathrm{em}}=-\frac{1}{4 e^{2}} F_{\mu \nu} F^{\mu \nu}-\frac{\theta}{16 \pi^{2}} F_{\mu \nu} \tilde{F}^{\mu \nu}$ shows that $e^{2} \equiv 1 / M_{\mathrm{b}}$ and $\theta \equiv 4 \pi^{2} N_{\mathrm{b}}$. Thus, $e$ and $\theta$ acquire time dependencies, as they are determined by the varying background $A_{\mathrm{b}}$ and $B_{\mathrm{b}}$.

The breakdown of Lorentz symmetry in our effective Lagrangian (2) is best exhibited by the resulting modified Maxwell equations:

$$
\frac{1}{e^{2}} \partial^{\mu} F_{\mu \nu}-\frac{2}{e^{3}}\left(\partial^{\mu} e\right) F_{\mu \nu}+\frac{1}{4 \pi^{2}}\left(\partial^{\mu} \theta\right) \tilde{F}_{\mu \nu}=0 \text {. }
$$


In our toy cosmology, the gradients of $e$ and $\theta$ are nonzero, approximately constant locally, and act as a nondynamical external background. Thus, the gradients select a preferred direction in local inertial frames violating Lorentz invariance. The term containing $\partial^{\mu} \theta$ can be identified with the $k_{A F}$ operator in the minimal SME. This term has recently received a lot of attention. ${ }^{28}$ For instance, it can lead to vacuum Cerenkov radiation. ${ }^{29}$

Next, we consider small localized excitations $\delta A$ and $\delta B$ of the scalar background in local inertial coordinates at the point $x_{0}$. With the ansatz $A(x)=A_{\mathrm{b}}(x)+\delta A(x)$ and $B(x)=B_{\mathrm{b}}(x)+\delta B(x)$ in the equations of motion for $A$ and $B$, we find the linearized equations

$$
\begin{aligned}
& 0=\left[\square-2 B^{\mu} \partial_{\mu}+2 m_{A}^{2} B_{\mathrm{b}}^{2}\right] \delta A-\left[2 A^{\mu} \partial_{\mu}-2 A^{\mu} B_{\mu}-4 m_{A}^{2} A_{\mathrm{b}} B_{\mathrm{b}}\right] \delta B, \\
& 0=\left[2 A^{\mu} \partial_{\mu}\right] \delta A+\left[\square-2 B^{\mu} \partial_{\mu}+6 m_{B}^{2} B_{\mathrm{b}}^{2}-A^{\mu} A_{\mu}+B^{\mu} B_{\mu}\right] \delta B
\end{aligned}
$$

where $A_{\mathrm{b}}, B_{\mathrm{b}}, A^{\mu} \equiv B_{\mathrm{b}}^{-1} \partial^{\mu} A_{\mathrm{b}}$, and $B^{\mu} \equiv B_{\mathrm{b}}^{-1} \partial^{\mu} B_{\mathrm{b}}$ are evaluated at $x_{0}$.

Equation (4) governs the propagation of $\delta A$ and $\delta B$ in our cosmological background. Note that $A^{\mu}$ and $B^{\mu}$ are external nondynamical vectors violating Lorentz symmetry. This result also applies to quantum theory: the excitations $\delta A$ and $\delta B$ would be seen as the effective particles corresponding to $A$ and $B$, so that such particles would break Lorentz invariance.

\section{Acknowledgments}

This work was funded by the Fundação Portuguesa para o Desenvolvimento.

\section{References}

1. R.R. Caldwell et al., Phys. Rev. Lett. 80, 1582 (1998); A. Masiero et al., Phys. Rev. D 61, 023504 (2000); Y. Fujii, Phys. Rev. D 62, 044011 (2000).

2. C. Armendáriz-Picón et al., Phys. Lett. B 458, 209 (1999).

3. A.H. Guth, Phys. Rev. D 23, 347 (1981).

4. V.A. Kostelecký et al., Phys. Rev. D 68, 123511 (2003).

5. O. Bertolami et al., Phys. Rev. D 69, 083513 (2004). R. Lehnert, grqc/0508122.

6. V.A. Kostelecký and R. Potting, Nucl. Phys. B 359, 545 (1991); Phys. Lett. B 381, 89 (1996); Phys. Rev. D 63, 046007 (2001); V.A. Kostelecký et al., Phys. Rev. Lett. 84, 4541 (2000).

7. G. Amelino-Camelia et al., Nature (London) 393, 763 (1998).

8. D. Sudarsky et al., Phys. Rev. D 68, 024010 (2003).

9. F.R. Klinkhamer, Nucl. Phys. B 578, 277 (2000).

10. J. Alfaro et al., Phys. Rev. Lett. 84, 2318 (2000).

11. S.M. Carroll et al., Phys. Rev. Lett. 87, 141601 (2001); A. Anisimov et al., Phys. Rev. D 65, 085032 (2002). 
12. D. Colladay and V.A. Kostelecký, Phys. Rev. D 55, 6760 (1997); 58, 116002 (1998); V.A. Kostelecký and R. Lehnert, Phys. Rev. D 63, 065008 (2001); V.A. Kostelecký, Phys. Rev. D 69, 105009 (2004).

13. OPAL Collaboration, R. Ackerstaff et al., Z. Phys. C 76, 401 (1997); DELPHI Collaboration, M. Feindt et al., preprint DELPHI 97-98 CONF 80 (1997); BELLE Collaboration, K. Abe et al., Phys. Rev. Lett. 86, 3228 (2001); BaBar Collaboration, B. Aubert et al., hep-ex/0303043.

14. V.A. Kostelecký and R. Potting, Phys. Rev. D 51, 3923 (1995).

15. D. Colladay and V.A. Kostelecký, Phys. Lett. B 344, 259 (1995); Phys. Rev. D 52, 6224 (1995); V.A. Kostelecký and R. Van Kooten, Phys. Rev. D 54, 5585 (1996); O. Bertolami et al., Phys. Lett. B 395, 178 (1997); N. Isgur et al., Phys. Lett. B 515, 333 (2001).

16. V.A. Kostelecký, Phys. Rev. Lett. 80, 1818 (1998); Phys. Rev. D 61, 016002 (2000); Phys. Rev. D 64, 076001 (2001).

17. D. Bear et al., Phys. Rev. Lett. 85, 5038 (2000); D.F. Phillips et al., Phys. Rev. D 63, 111101 (2001); M.A. Humphrey et al., Phys. Rev. A 68, 063807 (2003); Phys. Rev. A 62, 063405 (2000); V.A. Kostelecký and C.D. Lane, Phys. Rev. D 60, 116010 (1999); J. Math. Phys. 40, 6245 (1999).

18. R. Bluhm et al., Phys. Rev. Lett. 88, 090801 (2002).

19. F. Canè et al., Phys. Rev. Lett. 93, 230801 (2004).

20. H. Dehmelt et al., Phys. Rev. Lett. 83, 4694 (1999); R. Mittleman et al., Phys. Rev. Lett. 83, 2116 (1999); G. Gabrielse et al., Phys. Rev. Lett. 82, 3198 (1999); R. Bluhm et al., Phys. Rev. Lett. 82, 2254 (1999); Phys. Rev. Lett. 79, 1432 (1997); Phys. Rev. D 57, 3932 (1998).

21. L.-S. Hou et al., Phys. Rev. Lett. 90, 201101 (2003); R. Bluhm and V.A. Kostelecký, Phys. Rev. Lett. 84, 1381 (2000).

22. H. Müller et al., Phys. Rev. D 68, 116006 (2003); R. Lehnert, Phys. Rev. D 68, 085003 (2003); J. Math. Phys. 45, 3399 (2004).

23. S.M. Carroll et al., Phys. Rev. D 41, 1231 (1990); V.A. Kostelecký and M. Mewes, Phys. Rev. Lett. 87, 251304 (2001); J. Lipa et al., Phys. Rev. Lett. 90, 060403 (2003); Q. Bailey and V.A. Kostelecký, Phys. Rev. D 70, 076006 (2004).

24. V.W. Hughes et al., Phys. Rev. Lett. 87, 111804 (2001); R. Bluhm et al., Phys. Rev. Lett. 84, 1098 (2000); E.O. Iltan, JHEP 0306, 016 (2003).

25. V. Barger et al., Phys. Rev. Lett. 85, 5055 (2000); V.A. Kostelecký and M. Mewes, Phys. Rev. D 70, 031902 (2004).

26. D.L. Anderson et al., Phys. Rev. D 70, 016001 (2004).

27. E. Cremmer and B. Julia, Nucl. Phys. B 159, 141 (1979).

28. R. Jackiw and V.A. Kostelecký, Phys. Rev. Lett. 82, 3572 (1999); B. Altschul, Phys. Rev. D 69, 125009 (2004).

29. R. Lehnert and R. Potting, Phys. Rev. Lett. 93, 110402 (2004); Phys. Rev. D 70, 125010 (2004). 